\title{
A Novel Robust Tube Detection Filter for 3D Centerline Extraction ${ }^{\star}$
}

\author{
Thomas Pock, Reinhard Beichel, and Horst Bischof \\ Institute for Computer Graphics and Vision, Graz University of Technology, \\ Infeldgasse 16/2, A-8010 Graz, Austria \\ \{pock, beichel, bischof\}@icg.tu-graz.ac.at \\ http://www.icg.tu-graz.ac.at
}

\begin{abstract}
Centerline extraction of tubular structures such as blood vessels and airways in 3D volume data is of vital interest for applications involving registration, segmentation and surgical planing. In this paper, we propose a robust method for 3D centerline extraction of tubular structures. The method is based on a novel multiscale medialness function and additionally provides an accurate estimate of tubular radius. In contrast to other approaches, the method does not need any user selected thresholds and provides a high degree of robustness. For comparison and performance evaluation, we are using both synthetic images from a public database and a liver CT data set. Results show the advantages of the proposed method compared with the methods of Frangi et al. and Krissian et al.
\end{abstract}

\section{Introduction}

As a result of the development of modern volumetric imaging techniques like Computed Tomography (CT) and Magnetic Resonance Imaging (MRI), an increasing amount of anatomical and functional details of the human body can be acquired. However, with the benefit of higher spatial and radiometric resolution also the amount of generated data increases. For several applications like diagnosis or surgical planning, tubular structures like blood vessels are of interest. For example, the liver portal vein tree can be used for liver segment approximation, required for tumor resection planning 1. For 3D applications, such as visualization, segmentation or registration, the detection of vessel centerlines together with radius estimation is an useful preprocessing step. Manual centerline extraction is very time consuming, hence automatic and robust methods for tubular structures would greatly ease this process. In combination with $3 \mathrm{D}$ visualization methods, the analysis of the vessels can be substantially improved and simplified, as demonstrated by an Augmented Reality based liver surgery planning system, that has recently been developed 2 .

\footnotetext{
* This work was supported by the Austrian Science Fund (FWF) under the grant P17066-N04.
} 


\subsection{State of the Art}

Medialness functions are used to extract the medial axis of a structure by measuring the degree of belonging to its medial axis. A medialness function can be defined by the convolution product of a kernel $K(\mathbf{x}, \sigma)$ with a given image $I(\mathbf{x})$, where $\mathbf{x}=\left(x_{1}, x_{2}, x_{3}\right)^{T}$ is a point in $3 \mathrm{D}$ space and $\sigma$ denotes the scale of the measurement. Medialness functions can be classified according to two criteria:

(a) by the geometric location, where the measurements are made.

- Central medialness

- Offset medialness

(b) by the type of the filter kernel:

- Linear medialness

- Adaptive medialness

Frangi et al. 4 developed a vessel enhancement filter based on eigenvalue analysis of the scale space of the Hessian matrix. In terms of classification of medialness functions the vesselness enhancement filter can be classified as linear central medialness, because the eigenvalues are evaluated using a data independent filter kernel exclusively based on the central information. The scale space of the Hessian matrix is given by:

$$
\nabla^{2} I^{(\sigma)}(\mathbf{x})=\mathcal{H}^{(\sigma)}(\mathbf{x})=\sigma^{2 \gamma}\left[\frac{\partial^{2} I^{(\sigma)}}{\partial_{x_{i}} \partial_{x_{j}}}\right] .
$$

Let $\lambda_{1}, \lambda_{2}, \lambda_{3}$ and $\mathbf{v}_{1}, \mathbf{v}_{2}, \mathbf{v}_{3}$ be the eigenvalues and corresponding eigenvectors of $\mathcal{H}^{(\sigma)}(\mathbf{x})$ such that $\left|\lambda_{1}\right| \geq\left|\lambda_{2}\right| \geq\left|\lambda_{3}\right|$ and $\left|\mathbf{v}_{i}\right|=1$. The eigenvalues and eigenvectors correspond to the principal curvatures of the intensity function $I^{(\sigma)}$, which is the initial image convolved with a three-dimensional Gaussian kernel $G(\mathbf{x}, \sigma)=1 /\left(2 \pi \sigma^{2}\right)^{\frac{3}{2}} \exp \left(-\left(\mathbf{x}^{T} \mathbf{x}\right) /\left(2 \sigma^{2}\right)\right)$. The term $\sigma^{2 \gamma}$ in Eq. (1) is used for normalization of the second order derivatives, which ensures invariance under image rescaling 9 .

The dissimilarity measurement of Frangi et al. takes two geometric ratios into account. The first ratio addresses the deviation from a blob-like structure: $\mathcal{R}_{\mathcal{B}}=\left|\lambda_{1}\right| / \sqrt{\left|\lambda_{2} \lambda_{3}\right|}$. The second ratio is for distinguishing between plate-like and line-like structures and takes into account the two largest second order derivatives: $\mathcal{R}_{\mathcal{A}}=\left|\lambda_{2}\right| /\left|\lambda_{3}\right|$. In order to diminish the response of the background pixels, the Frobenius norm of the Hessian matix is used to define the measure of second order structureness: $\mathcal{S}=\sqrt{\lambda_{1}^{2}+\lambda_{2}^{2}+\lambda_{3}^{2}}$. Using these three measures a vessel likelihood function is defined:

$$
\mathcal{V}_{0}(\sigma)= \begin{cases}0 & \text { if } \quad \lambda_{2}>0 \text { or } \lambda_{3}>0 \\ \left(1-e^{-\frac{\mathcal{R}_{\mathcal{A}}^{2}}{2 \alpha^{2}}}\right)\left(e^{-\frac{\mathcal{R}_{\mathcal{B}}^{2}}{2 \beta^{2}}}\right)\left(1-e^{-\frac{\mathcal{S}^{2}}{2 c^{2}}}\right) \text { otherwise }\end{cases}
$$

where $\alpha, \beta$ and $c$ are thresholds to control the sensitivity of the filter to the measures $\mathcal{R}_{\mathcal{A}}, \mathcal{R}_{\mathcal{B}}$ and $\mathcal{S}$. The filter is applied at multiple scales and the maximum response across scales is selected. 


$$
\mathcal{V}=\max _{\sigma_{\min } \leq \sigma \leq \sigma_{\max }}\left\{\mathcal{V}_{0}(\sigma)\right\}
$$

Krissian et al. 8 developed an adaptive offset medialness function for 3D brain vessel segmentation. The function is said to be adaptive, because the orientation of the filter kernel is locally adapted by the eigenvectors $\mathbf{v}_{1}$ and $\mathbf{v}_{2}$, which correspond to the two major principial curvatures of the Hessian matrix. Furthermore, the function is classified as an offset medialness function, because the function measures contour information at points equidistant to the center. The medialness function is given by:

$$
R(\mathbf{x}, \sigma, \theta)=\frac{1}{2 \pi} \int_{\alpha=0}^{2 \pi}-\sigma^{\gamma} \nabla I^{(\sigma)}\left(\mathbf{x}+\theta \sigma \mathbf{v}_{\alpha}\right) \cdot \mathbf{v}_{\alpha} d \alpha
$$

where $\mathbf{v}_{\alpha}=\cos (\alpha) \mathbf{v}_{1}+\sin (\alpha) \mathbf{v}_{2}$ and $\alpha$ is the angle of the rotating phasor $\mathbf{v}_{\alpha}$. Thus, the function measures the gradient of the smoothed image along a circle with radius $\theta \sigma$ in the plane defined by $\mathbf{v}_{1}$ and $\mathbf{v}_{2}$. In analogy to Eq. (1), the term $\sigma^{\gamma}$ is used to normalize the first order derivatives.

The authors use a cylindrical model with Gaussian cross section to analytically compute the value of the proportionality constant $\theta$, at which the medialness function gives a maximal response. Having set $\gamma$ to the value 1 , the proportionality constant equals $\theta=\sqrt{3}$. Furthermore, for radius estimation, the authors derive a relation between the radius of the vessel $\sigma_{0}$ and the scale $\sigma_{\max }$ at which it is detected:

$$
\theta \sqrt{\sigma_{\max }^{2}}=\sqrt{\sigma_{0}^{2}+\sigma_{\max }^{2}} \Rightarrow \sigma_{0}=\sqrt{2} \sigma_{\max } .
$$

Similarly to Frangi's method, the function is applied at different scales and the maximum response is selected.

$$
R_{\text {multi }}(\mathbf{x}, \theta)=\max _{\sigma_{\min } \leq \sigma \leq \sigma_{\max }}\{R(\mathbf{x}, \sigma, \theta)\} .
$$

In $[5$, two weighting factors were introduced to increase the robustness of the method, when applied to 3D Ultrasound images of the Aorta.

Once the mutiscale medialness response is generated, the centerlines can be extracted using the method of Pizer et al. 11]. Considering the multiscale medialness response, it is obvious that the local maxima correspond to the medial axes of the tubes. Therefore, if all the local maxima are located at central points of the tubes, local maxima extraction is equivalent to centerline detection. The characterization of local extrema is based on the properties of the Hessian matrix. A three dimensional image point $\mathbf{x}$ is considered to be locally maximal in the multiscale medialness response $R_{\text {mult }}$, if the following condition is satisfied:

$$
R_{\text {multi }}(\mathbf{x}) \geq R_{\text {multi }}\left(\mathbf{x} \pm \mathbf{v}_{1}\right) \quad \text { and } \quad R_{\text {multi }}(\mathbf{x}) \geq R_{\text {multi }}\left(\mathbf{x} \pm \mathbf{v}_{2}\right) .
$$

The eigenvectors $\mathbf{v}_{1}, \mathbf{v}_{2}$ correspond to the two major components of the principal curvatures of the Hessian matrix, obtained from the scale that provides the maximum response. In order to obtain a skeleton like representation of the centerlines, the result of the local maxima extraction is thinned by deleting the simple points 10. 


\subsection{Contribution}

In this paper, a novel robust tube detection filter for 3D centerline extraction is presented. The filter is based on an adaptive multiscale medialness function, which combines offset and central information. The function measures boundariness along a circle in the plane defined by the two largest principial curvatures of the Hessian matrix. We further propose a weighting function that takes the symmetry of the tube into account. Thus, responses resulting from isolated structures or high intensity variations are rejected and no background subtraction is needed. To avoid the need for user-selected thresholds, the function includes an adaptive threshold, which is based on a central medianless function and allows better discrimination between tube and non-tube structures. In contrast to the method of Krissian et al., we do not use the same scale for computing both the image gradient and the Hessian matrix. Therefore, our method is less dependent on the vessel model and provides accurate radius estimates. In order to compare our method to the methods of Frangi et al. and Krissian et al., we are using both synthetic images taken from a public database [6,7,8 and a liver CT data set. The results show that our method provides a higher accuracy of the centerline and the radius estimate.

\section{Method}

In 8 the proportional parameter $\theta$ is used, to define a linear relation between the radius of the tube and the scale space parameter $\sigma$ (Eq.(4)). With this relation, their method is very inflexible especially in providing radius estimates for nonGaussian tube models. In order to achieve model independence we are using two different scale spaces for computing the Hessian matrix and the boundariness and define a more flexible relation given by

$$
\sigma_{\mathbf{B}}=\sigma_{\mathcal{H}}^{\eta},
$$

where $\eta$ depends on the amount of noise in the image and ranges between $[0,1]$. Thus, the Hessian and boundariness scale spaces are defined by:

$$
\mathcal{H}(\mathbf{x})=\sigma_{\mathcal{H}}^{2 \gamma}\left[\frac{\left.\partial^{2} I_{\mathcal{H}}\right)}{\partial_{x_{i}} \partial_{x_{j}}}\right] \quad \text { and } \quad \mathbf{B}(\mathbf{x})=\sigma_{\mathbf{B}}^{\gamma} \nabla I^{\left(\sigma_{\mathbf{B}}\right)}(\mathbf{x}) .
$$

The boundariness can be represented by $b(\mathbf{x})=|\mathbf{B}(\mathbf{x})|$ and $\mathbf{g}(\mathbf{x})=\mathbf{B}(\mathbf{x}) /|\mathbf{B}(\mathbf{x})|$, which are the magnitude and the direction of the gradient.

Based on these scale spaces, the method consists of the following steps: First, the initial medialness is computed, using contour information of the boundariness scale space and the filter kernel is locally adapted by means of the Hessian scale space. Second, the initial medialness is weighted by a function that takes the symmetry of the structure into account. Third, a gradient based central medialness function is used to obtain an adaptive threshold. Finally, the function is evaluated at different scales and local maxima are extracted. 


\subsection{Initial Medialness}

The initial medialness is given by averaging the contribution of boundariness around a circle of radius $r$ in the plane defined by the eigenvectors $\mathbf{v}_{1}$ and $\mathbf{v}_{2}$, to the point $\mathbf{x}$ in the medialness space:

$$
R_{0}^{+}(\mathbf{x}, r)=\frac{1}{N} \sum_{i=0}^{N-1} b\left(\mathbf{x}+r \mathbf{v}_{\alpha_{i}}\right) c_{i}^{n}
$$

where $N$ is the number of samples and is calculated by $N=\lfloor 2 \pi \sigma+1\rfloor$ and $\alpha_{i}=(2 \pi i) / N$, respectively. The circularity, $c_{i}$, measures the contribution of the boundariness in radial direction $\mathbf{v}_{\alpha_{i}}=\cos \left(\alpha_{i}\right) \mathbf{v}_{1}+\sin \left(\alpha_{i}\right) \mathbf{v}_{2}$ and is additionally constrainted by the circularity parameter $n$. The choice of the circularity parameter is not very critical, $n=2$ being applicable for most images.

$$
c_{i}=\left\{\begin{array}{ll}
-\mathbf{g}\left(\mathbf{x}+r \mathbf{v}_{\alpha_{i}}\right) \cdot \mathbf{v}_{\alpha_{i}} & \text { if }-\mathbf{g}\left(\mathbf{x}+r \mathbf{v}_{\alpha_{i}}\right) \cdot \mathbf{v}_{\alpha_{i}}>0 \\
0 & \text { otherwise }
\end{array} .\right.
$$

\subsection{Symmetry Confidence}

Eq. (10) also produces responses for isolated edges and non-tube-like structures of high intensity variation. In order to increase the selectivity of the detection, a criterion that takes the symmetry property of the object into account, is introduced. Defining the $i^{\text {th }}$ boundariness sample by $b_{i}=b\left(\mathbf{x}+r \mathbf{v}_{\alpha_{i}}\right) c_{i}^{n}$, Eq. (10) can be rewritten as

$$
R_{0}^{+}(\mathbf{x}, r)=\frac{1}{N} \sum_{i=0}^{N-1} b_{i}
$$

Considering the distribution of the values $b_{i}$, it is obvious that symmetric structures have a low variance compared to non-symmetric structures. We introduce the variance of the boundariness samples:

$$
\left.s^{2}(\mathbf{x}, r)=\frac{1}{N} \sum_{i=0}^{N-1}\left(b_{i}-\bar{b}\right)\right)^{2},
$$

where $\bar{b}=R_{0}^{+}(\mathbf{x}, r)$ is the mean boundariness. To quantify the homogeneity of the boundariness along the circle, the initial medialness is weighted by the symmetry confidence

$$
\mathcal{S}(\mathbf{x}, r)=1-\frac{s^{2}(\mathbf{x}, r)}{\bar{b}^{2}} .
$$

For circular symmetric structures, $s^{2}$ is very low compared to $\bar{b}^{2}$ and hence, $\mathrm{S}(\mathbf{x}$, $\mathrm{r})$ is approximately one. The larger $s^{2}$, the smaller the value $\mathrm{S}(\mathbf{x}, \mathrm{r})$, which results in a reduction in the response to non circular symmetric structures. Thus, the symmetry constrainted medialness is defined as:

$$
R^{+}(\mathbf{x}, r)=R_{0}^{+}(\mathbf{x}, r) \mathcal{S}(\mathbf{x}, r)
$$




\subsection{Adaptive Threshold}

One problem associated with medialness functions is to find an appropriate threshold to define a minimum response, needed to reject noise and outliers. Considering an arbitrary symmetric cross section profile of a tubular structure, one can notice that the magnitude of the image gradient vanishes at the tube's centerline. Therefore, an adaptive threshold is obtained by the simple fact that, for the tube's center, the medialness must be larger than the magnitude of the center gradient. For this purpose, we introduce the norm of the center gradient

$$
R^{-}(\mathbf{x}, r)=\sigma_{\mathcal{H}}^{\gamma}\left|\nabla I^{\left(\sigma_{\mathcal{H}}\right)}(\mathbf{x})\right|,
$$

which can be classified as a central medialness function. Here, we use the same scale $\sigma_{\mathcal{H}}$, to compute the Hessian matrix and the center gradient. Since both features are central ones, the information of the whole structure should be included to provide accurate estimates. Considering a tube of radius $r^{\prime}$, equation (10) is maximized, if the scale parameter $r$ is equal to $r^{\prime}$. At the same time, the Hessian matrix and the center gradient are computed most stably, if the characteristic width of the Gaussian convolution kernel appproximately corresponds to the radius of the tube. Hence, we set $\sigma_{\mathcal{H}}=r$. The final medialness is obtained by combining the offset medialness $R^{+}(\mathbf{x}, r)$ and the center medialness $R^{-}(\mathbf{x}, r)$ :

$$
R(\mathbf{x}, r)=\left\{\begin{array}{ll}
R^{+}(\mathbf{x}, r)-R^{-}(\mathbf{x}, r) & \text { if } R^{+}(\mathbf{x}, r)>R^{-}(\mathbf{x}, r) \\
0 & \text { otherwise }
\end{array} .\right.
$$

\subsection{Multiscale Analysis}

To take into account the varying size of the vessels, the medialness function $R(\mathbf{x}, r)$ is evaluated at different radii $r$. The multiscale medialness response is obtained by selecting the maximum response over a range of different radii defined by $r_{\min }$ and $r_{\max }$ :

$$
R_{\text {multi }}(\mathbf{x})=\max _{r_{\text {min }} \leq r \leq r_{\text {max }}}\{R(\mathbf{x}, r)\} .
$$

The scale $r$ at which the tube is detected is used to determine the radius of the vessels. In [8, the authors showed how to use the maximum response of their multiscale medialness function to estimate the radius of Gaussian tube models. They also showed, that in the case of bar-like cross sections, the radius estimation fails. However, the use of two separate scale spaces enables tube model independence. In our approach, the accuracy of the radius estimate is only influenced by the amount of image noise.

\section{$3 \quad$ Experimental Results}

\subsection{Synthetic Images}

We are using four characteristic 3D synthetic images (see Fig. 11) from the public data base of Krissian et al. 6, 7, 8, Fig. 1] shows the computed centerlines of the 


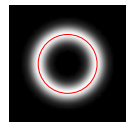

(a)

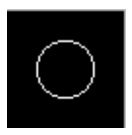

(e)

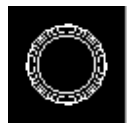

(i)

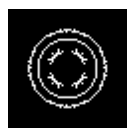

$(\mathrm{m})$

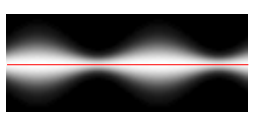

(b)

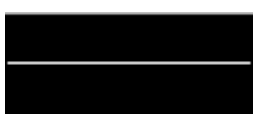

(f)

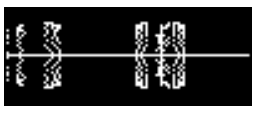

(j)

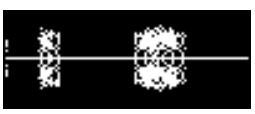

(n)

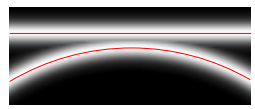

(c)

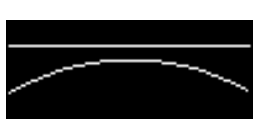

(g)

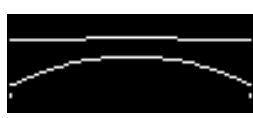

(k)

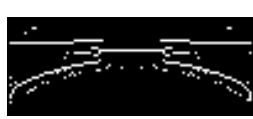

(o)

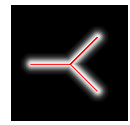

(d)

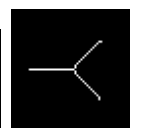

(h)

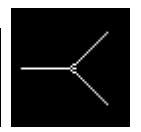

(1)

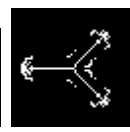

(p)

Fig. 1. Maximum Intensity Projection (MIP) of the testimages used in evaluation. (a) Tore, (b) Varying Cylinder, (c) Tangent Vessels and (d) Y-Junction. Extracted centerlines: (e)-(h) proposed method, (i)-(l) method of Frangi and (m)-(p) method of Krissian

Table 1. Quantitative analysis of centerline accuracy

\begin{tabular}{|c|c|c|c|c|c|c|c|c|c|c|c|c|c|}
\hline & \multicolumn{3}{|c|}{ Tore } & \multicolumn{4}{c|}{ Varying Radius } & \multicolumn{3}{c|}{ Tangent Vessels } & \multicolumn{3}{c|}{ Y-Junction } \\
\hline & $R_{\text {pos }}$ & $\mu_{\text {pos }}$ & $R_{\text {neg }}$ & $R_{\text {pos }}$ & $\mu_{\text {pos }}$ & $R_{\text {neg }}$ & $R_{\text {pos }}$ & $\mu_{\text {pos }}$ & $R_{\text {neg }}$ & $R_{\text {pos }}$ & $\mu_{\text {pos }}$ & $R_{\text {neg }}$ \\
\hline Proposed & $\mathbf{0 . 0 0}$ & $\mathbf{0 . 0 0}$ & $\mathbf{0 . 0 0}$ & $\mathbf{0 . 0 0}$ & $\mathbf{0 . 0 0}$ & $\mathbf{0 . 0 0}$ & $\mathbf{0 . 0 0}$ & $\mathbf{0 . 0 0}$ & $\mathbf{0 . 0 0}$ & $\mathbf{0 . 1 0}$ & $\mathbf{1 . 0 0}$ & $\mathbf{0 . 0 4}$ \\
\hline Frangi et al. & 5.98 & 5.21 & 0.00 & 7.81 & 11.78 & 0.00 & 0.55 & 1.36 & 0.49 & 0.50 & 3.08 & 0.06 \\
\hline Krissian et al. & 5.62 & 4.58 & 0.33 & 11.12 & 9.54 & 0.00 & 1.03 & 2.96 & 0.54 & 5.40 & 4.66 & 0.04 \\
\hline
\end{tabular}

proposed method, the other methods tested. It can be seen that by our method, the centerlines are extracted very accurately. The main reason is, that in regions of high image gradients, the medialness response is eliminated by the adaptive threshold. In addition, the approaches by Frangi and Krissian require thresholds in order to achieve better results.

For quantification, we define three measures, $R_{\text {pos }}, \mu_{\text {pos }}$ and $R_{n e g}$, which are the rate of falsely positive centerline voxels, the mean distance in voxel of the falsely centerline voxels to the real centerline, and the rate of falsely negative centerline voxels detected. Table 1 details the quantitative anlysis of the extracted centerlines. The centerlines of the models Tore, Varying Cylinder 


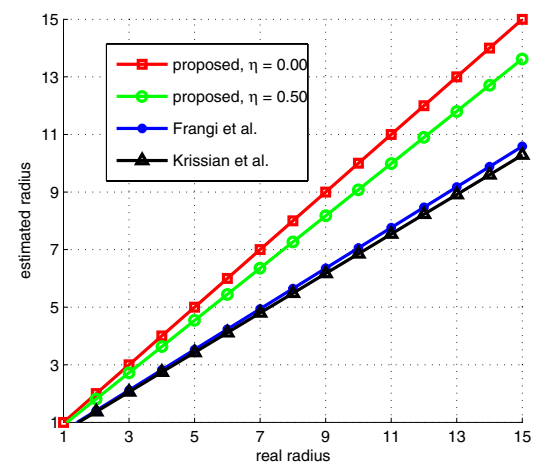

Fig. 2. Results of radius estimation for bar-like tubes of radius $1-15$

and Tangent Vessels were accurately extracted. The centerline of the model $Y$ Junction containes some corrupted voxels, but the mean deviation of the false positive voxels is very small ( 1 voxel). The following parameters were used: $\eta=0.00$ and $n=2$. The parameters of the other approaches were set to standard values as descibed in 4 and 8 .

\subsection{Performance of Radius Estimation}

In real images the intensity variation inside a contrast enhanced vessel is not very high, but the vessel borders are affected by the partial volume effect. Therefore, small vessels are approximated well by a Gaussian model, but big ones are better modeled by a bar-like cross section convolved with a Gaussian Kernel with a small standard deviation. For evaluation of the radius estimation we use bar-like tube models of radius $1-15$ voxels, convolved with a Gaussian smoothing kernel of standard deviation 1 voxel. Furthermore, we use different values of the noise parameter $\eta$, to demonstrate how this influences the accuracy of the radius estimation. Fig. 2 shows the error of the radius estimation of the proposed method and the methods of Frangi et al. and Krissian et al. The small tubes approximately match the Gaussian model and thus, the methods of Frangi and Krissian do not result in large errors. The more, the tubes approximate the bar-like model, the greater is the error associated with these methods. However, the radius estimation of our method does not depend on the specific type of the tube model.

\subsection{Real Data Sets}

We used a liver CT data set which was acquired from routine clinical scan with a voxel size of $0.55 \times 0.55 \times 2.00 \mathrm{~mm}^{3}$ and a volume size of $512 \times 512 \times 97$ voxels. The anisotropic voxels were converted into isotropic voxels using sinc interpolation [3. For computational speedup, two thresholds $t_{\min }$ and $t_{\max }$ were chosen to define the intensity value range of interest and the computation was limited to regions inside the liver. The following parameters were used: $\eta=$ 


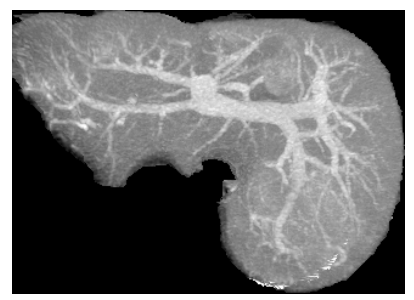

(a)

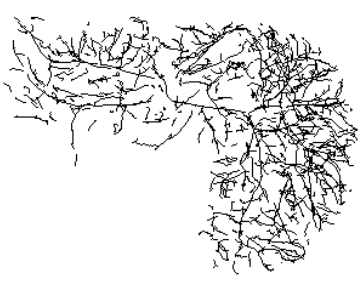

(b)

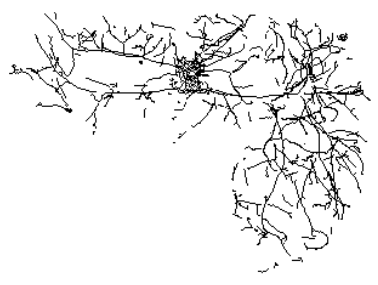

(c)

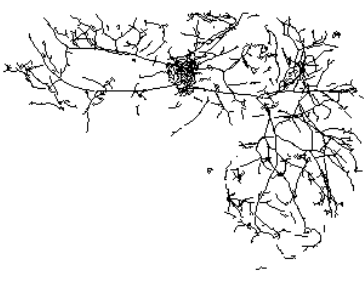

(d)

Fig. 3. Extracted centerlines of a liver portal vein tree. (a) MIP of initial image. Extracted centerlines of (b) the proposed method, (c) method of Frangi and (d) method of Krissian

$0.5, n=2, r_{\min }=1$ and $r_{\max }=15$. In order to improve the results of the methods of Frangi et al. and Krissian et al., the background voxels defined by $I(\mathbf{x})<t_{\text {min }}$ were eliminated by setting them to the value $t_{\min }$. Furthermore, the medialness responses of Frangi and Krissian were thresholded, using the values: $t_{F}=0.05$ and $t_{K}=7.00$. For both these methods standard parameter settings were used, as described in [4] and 8]. Fig. 3] shows a comparison of the extracted centerlines of a liver portal vein tree for all the different methods. It can be seen, that the complexity and the quality of the centerline extracted by the proposed method is considerably better, than those from the other approaches. In particular, they were less effective at detecting the centerlines of large vessels. Due to intensiy variations inside large vessels, incorrect local maxima in the multiscale medialness response may emerge and result in erroneous centerline pieces. Another disadvantage of the methods of Frangi et al. and Krissian et al. is the need to threshold the medialness response. On one hand, a certain degree of user interaction is necessary to select the threshold, while on the other hand, small vessels providing only a low medialness response are rejected.

\section{Conclusion and Future Work}

In this paper, a novel tube detection filter for 3D centerline extraction was presented. In order to compare our method with the methods of Frangi et al. and 
Krissian et al., we used synthetic images and a liver CT data set. The results show the robustness of the method as well as its ability to provide model-independent accurate radius estimates.

Future work will concentrate mainly on special cases of data sets acquired during routine clinical scans with a lot of noise and low contrast between tubes and background.

\section{References}

1. R. Beichel, T. Pock, C. Janko, R. Zotter, B. Reitinger, A. Bornik, K. Palágyi, E. Sorantin, G. Werkgartner, H. Bischof, and M. Sonka. Liver segment approximation in CT data for surgical resection planning. In Proc. of SPIE, volume 5370, pages 1435-1446, May 2004.

2. A. Bornik, R. Beichel, B. Reitinger, G. Gotschuli, E. Sorantin, F. Leberl, and M. Sonka. Computer aided liver surgery planning: An augmented reality approach. In SPIE Imaging 2003: Visualization, Image-Guided Procedures and Display, pages 395-405, February 2003.

3. Y. Du, D. Parker, and W. Davis. Reduction of partial-volume artifacts with zerofilled interpolation in three-dimensional $\mathrm{mr}$ angiography,. Journal of Magnetic Resonance Imaging, 4(5):733-741, 1995.

4. A. Frangi. Three-Dimensional Model-Based Analysis of Vascular and Cardiac Images. PhD thesis, University Medical Center Utrecht, Netherlands, 2001.

5. K. Krissian, J. Ellsmere, K. Vosburgh, R. Kikinis, and C. F. Westin. Multiscale segmentation of the aorta in 3D ultrasound images. In 25th Annual Int. Conf. of the IEEE Engineering in Medicine and Biology Society, EMBS, pages 638-641, Cancun Mexico, 2003.

6. K. Krissian and G. Farneback. Synthetical test images for vascular segmentation algorithms. http://lmi.bwh.harvard.edu/research/vascular/SyntheticVessels/ SyntheticVesselImages.html.

7. K. Krissian and G. Farneback. Techniques in the enhancement of 3D angiograms and their application. To appear in Book Chapter.

8. K. Krissian, G. Malandain, N. Ayache, R. Vaillant, and Y. Trousset. Model-based detection of tubular structures in 3D images. Computer Vision and Image Understanding, 80(2):130-171, 2000.

9. Tony Lindeberg and Daniel Fagerstrom. Scale-space with casual time direction. In ECCV (1), pages 229-240, 1996.

10. G. Malandain, G. Bertrand, and N. Ayache. Topological segmentation of discrete surfaces. International Journal of Computer Vision, 10(2):183-197, 1993.

11. S. M. Pizer, K. Siddiqi, G. Sźekely, J. N. Damon, and S. W. Zucker. Multiscale medial loci and their properties. International Journal of Computer Vision, 55(2/3):155-179, 2003. 